Research Article

\title{
Analyses of Delamination in Functionally Graded Multilayered Beams
}

\author{
V. I. Rizov
}

Department of Technical Mechanics,University of Architecture, Civil Engineering and Geodesy, 1 Chr. Smirnensky blvd.1046, Sofia, Bulgaria

Received 7 July 2017; Accepted 15 December 2017

\begin{abstract}
Delamination fracture in three dimensional functionally graded multilayered beams loaded by bending moments and axial forces was analyzed. The beams can have an arbitrary number of layers of different thicknesses and material properties. In each layer, the material is functionally graded in the width, thickness and length directions. A delamination crack is located arbitrary along the height of the beam cross-section. Linear-elastic behaviour of the material was assumed. The bending moments and axial forces in the beam cross-sections ahead and behind the crack front were used to obtain a closed form analytical solution for the strain energy release rate which was applied to investigate the fracture in the functionally graded multilayered End Loaded Split (ELS) beam configuration. The strain energy release rate in the ELS was analyzed also by considering the beam strain energy for verification. Parametric studies were carried-out to evaluate the effect of material gradient and crack location on the fracture
\end{abstract}

Keywords: Functionally graded multilayered structure, Fracture, Linear-elastic material

\section{Introduction}

Functionally graded materials have been used extensively in various engineering applications in aeronautics, microelectronics, thermal protective systems, optics and biomechanics for the last thirty years [1], [2], [3], [4], [5], [6]. Functionally graded materials are novel composites whose composition changes continuously within the solid. In this way, concentrations of stresses between different constituent materials are avoided in contrast to layered composites [7], [8], [9]. By variation of their properties with specific gradients along spatial coordinates, functionally graded materials can be designed to satisfy different requirements in different parts of a member. The functionality and reliability of structural members and components made of these novel materials are strongly influenced by their fracture behaviour. Therefore, various fracture analyses of functionally graded materials and structures have been performed by many researchers around the world [10], [11], [12], [13], [14], [15]. Although significant efforts have been devoted to study fracture of these materials, still there are problems which have not been analyzed sufficiently. One of these problems is the delamination in three-dimensional functionally graded multilayered structural members and components.

Therefore, the main goal of this paper was to analyze the delamination fracture in three-dimensional functionally graded multilayered beams loaded by bending moments and axial forces. The fracture was analyzed in terms of the strain energy release rate assuming linear-elastic behaviour of the material. The common solution derived was used to obtain

\footnotetext{
*E-mail address: v_rizov_the@uacg.bg

ISSN: $1791-2377$ C 2017 Eastern Macedonia and Thrace Institute of Technology. All rights reserved.

doi:10.25103/jestr.106.15
}

the strain energy release rate in the functionally graded multilayered ELS beam. The dependences of strain energy release rate on the material gradient and crack location were evaluated.

\section{Determination of the strain energy release rate}

Linear-elastic functionally graded multilayered beam configurations with a delamination crack located arbitrarily between were studied. The beams are loaded by bending moments and axial forces. A perfect adhesion between layers was assumed. A beam portion with the delamination crack front is shown schematically in Fig. 1. The beam width at the delamination level is $b_{S}$. The beam height is $2 h$.

The lower and upper crack arm thicknesses are $h_{1}$ and $h_{2}$, respectively. The beam cross-section is symmetric with respect to the $z$-axis. The beam is built up by $n$ horizontal layers. In each layer, the material is functionally graded in length, width and thickness directions. Thus, the modulus of elasticity in the $i$-th layer, $E_{i}$, is a function of $x, y$ and $z$, i.e. $E_{i}=E_{i}(x, y, z)$, where $i=1,2, \ldots, n$.

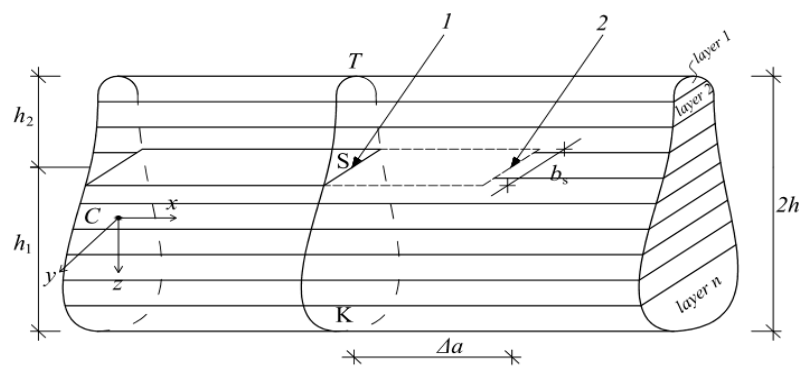

Fig. 1. Beam portion with the delamination crack front $(1-$ front position before the increase of crack, 2 - front position after the increase of crack). 
The dealamination was analyzed in terms of the strain energy release rate by using methods of linear-elastic fracture mechanics. In order to derive the strain energy release rate, a small increase, $\Delta A_{a}$, of the crack area was assumed (the external load was kept constant). The strain energy release rate, $G$, for linear-elastic materials can be expressed as [16]

$G=\frac{\Delta U}{\Delta A_{a}}$

where

$\Delta A_{a}=b_{s} \Delta a$

In Eq. 1, $\Delta U$ is the change of strain energy that can be written as

$\Delta U=U_{a}-U_{b}$,

where $U_{b}$ and $U_{a}$ are the strain energies before and after the increase of crack area, respectively. By combining of Eqs. 1, 2 and 3, $G$ can be written as

$$
G=\frac{U_{a}-U_{b}}{b_{s} \Delta a} .
$$

Eq. 4 was used to obtain the strain energy release rate in the present paper.
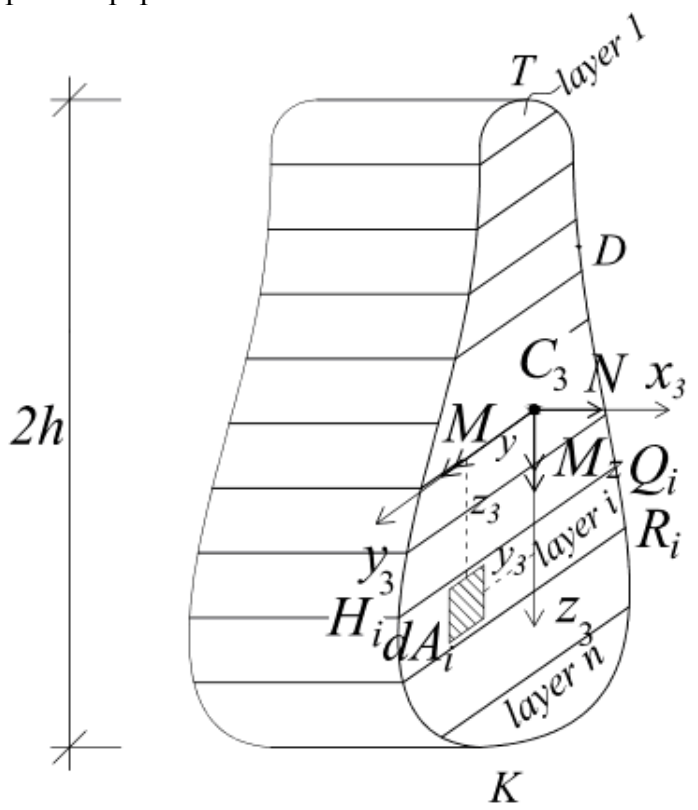

Fig. 2. Beam cross-section, $T K$, before the increase of crack.

The beam cross-section before the crack increase is shown in Fig. 2. The strain energy before the crack increase was written as

$U_{b}=\Delta a \sum_{i=1}^{i=n} \iint_{\left(A_{i}\right)} u_{0 i} d A_{i}$,

where $A_{i}$ is the are of $i$-th layer. The strain energy density, $u_{0 i}=\frac{\sigma_{i}^{2}}{2 E_{i}}$

The stress distribution in the $i$-th layer was obtained by the Hooke's law

$\sigma_{i}=E_{i} \varepsilon$,

where $\varepsilon$ is the strain distribution.

It was assumed that in the $i$-th layer $E_{H_{i}}, E_{Q_{i}}$ and $E_{R_{i}}$ are the values of modulus of elasticity in points $H_{i}, Q_{i}$ and $R_{i}$, respectively (Fig. 2). The present analysis was carriedout assuming linear variation of the modulus of elasticity, $E_{i}$, along the width and thickness of each layer. Therefore, the distribution of modulus of elasticity in the $i$-th layer was expressed in a function of $y_{3}$ and $z_{3}$ through $E_{H_{i}}, E_{Q_{i}}$ and $E_{R_{i}}$ by using the following equation [17] of a plane that passes via three points of coordinates $\left(E_{H_{i}}, y_{3 H_{i}}, z_{3 H_{i}}\right)$, $\left(E_{Q_{i}}, y_{3 Q_{i}}, z_{3 Q_{i}}\right)$ and $\left(E_{R_{i}}, y_{3 R_{i}}, z_{3 R_{i}}\right)$ :

$\left|\begin{array}{cccc}E_{i} & y_{3} & z_{3} & 1 \\ E_{H_{i}} & y_{3 H_{i}} & z_{3 H_{i}} & 1 \\ E_{Q_{i}} & y_{3 Q_{i}} & z_{3 Q_{i}} & 1 \\ E_{R_{i}} & y_{3 R_{i}} & z_{3 R_{i}} & 1\end{array}\right|=0, i=1,2, \ldots, n$

It should be noted that $E_{H_{i}}, E_{Q_{i}}$ and $E_{R_{i}}$ are functions of $x$, since they vary continuously along the beam length.

The strain distribution was analyzed assuming validity of the Bernoulli's hypothesis for plane sections, since the span to height ratio of beams considered is large. It should be mentioned that the Bernoulli's hypothesis has been frequently used in fracture studies of functionally graded materials $[13,14]$. Concerning the application of Bernoulli's hypothesis in the present paper, it can also be noted that due to the fact that the multilayered functionally graded beam is under a combination of bending moments and an axial force (Fig. 2), only the longitudinal strain, $\mathrm{x} \varepsilon$, is non-zero. Thus, the small strain compatibility equations indicate that, $\varepsilon$ is distributed linearly in beam cross-section. Therefore, the strain, $\varepsilon$, was expressed in a function of $y_{3}$ and $z_{3}$ by using the strains, $\varepsilon_{T}, \varepsilon_{D}$ and $\varepsilon_{K}$, in points $T, D$ and $K$. For this purpose, the following equation of a plane [17] that passes though points, $\left(\varepsilon_{T}, y_{3 T}, z_{3 T}\right), \quad\left(\varepsilon_{D}, y_{3 D}, z_{3 D}\right)$ and $\left(\varepsilon_{K}, y_{3 K}, z_{3 K}\right)$, was applied:

$\left|\begin{array}{llll}\varepsilon & y_{3} & z_{3} & 1 \\ \varepsilon_{T} & y_{3 T} & z_{3 T} & 1 \\ \varepsilon_{D} & y_{3 D} & z_{3 D} & 1 \\ \varepsilon_{K} & y_{3 K} & z_{3 K} & 1\end{array}\right|=0$.

$u_{0 i}$, in the $i$-th layer was expressed as 
The strains, $\varepsilon_{T}, \varepsilon_{D}$ and $\varepsilon_{K}$, can be determined from the following equations for equilibrium of the beam crosssections (Fig. 2):

$$
\begin{aligned}
& N=\sum_{i=1}^{i=n} \iint_{\left.A_{i}\right)} \sigma_{i} d A_{i}, \\
& M_{y}=\sum_{i=1}^{i=n} \iint_{\left(A_{i}\right)} \sigma_{i} z_{3} d A_{i}, \\
& M_{z}=\sum_{i=1}^{i=n_{L}} \iint_{\left(A_{i}\right)} \sigma_{i} y_{3} d A_{i},
\end{aligned}
$$

where $N, M_{y}$ and $M_{z}$ are the axial force and the bending moments for $y_{3}$ and $z_{3}$ axes, respectively (Fig. 2). The stress, $\sigma_{i}$, in Eqs. 10, 11 and 12 can be expressed by using the Hooke's law Eq.7, where the moduli of elasticity, $E_{i}$, and the strain, $\varepsilon$, are determined by Eqs. 8 and 9 , respectively. Then Eqs. 10,11 and 12 have to be solved with respect to $r_{1}, r_{2}$ and $r_{3}$ for a particular form of the beam cross-section. The results of strain analysis can be applied to calculate the strain energy density that is used to determine the strain energy, $U_{b}$.
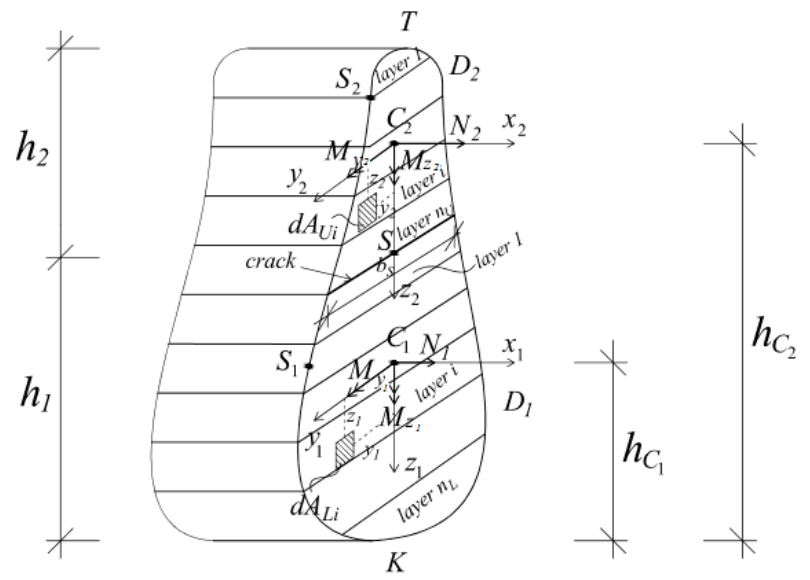

Fig. 3. Beam cross-section, $T K$, after the increase of crack.

The strain energy, $U_{a}$, after the crack increase was found as

$U_{a}=U_{a_{1}}+U_{a_{2}}$

where an $U_{a_{1}} \mathrm{~d} U_{a_{2}}$ are the strain energies in the lower and upper crack arm, respectively. The geometry and loading of cross-section of crack arms after the increase of crack are shown schematically in Fig. 3. The lower and upper crack arm thicknesses are $h_{1}$ and $h_{2}$, respectively. The number of layers in the lower and upper crack arm are $n_{L}$ and $n_{U}$, respectively. The strain energy in the lower crack arm was written as
$U_{a_{1}}=\Delta a \sum_{i=1}^{i=n_{L}} \iint_{\left(A_{L i}\right)} u_{01 i} d A_{L i}$

where $u_{01 i}$ and $A_{L i}$ are the strain energy density and the area of $i$-th layer of lower crack arm. The strain distribution, $\varepsilon$, in the lower crack arm was analyzed by Eq. 9. For this purpose, $y_{3}$ and $z_{3}$ were replaced with $y_{1}$ and $z_{1}$, respectively (the axes $y_{1}$ and $z_{1}$ are shown in Fig. 3). Also, $\varepsilon_{T}$ and $\varepsilon_{D}$ were replaced with $\varepsilon_{S_{1}}$ and $\varepsilon_{D_{1}}$, respectively. The strains, $\varepsilon_{S_{1}}$, $\varepsilon_{D_{1}}$ and $\varepsilon_{K}$ were determined from Eqs. 10, 11 and 12. For this purpose, $N, M_{y}, M_{z}, n, y_{3}, z_{3}$ and $A_{i}$ were replaced with $N_{1}, M_{y_{1}}, M_{z_{1}}, n_{L}, y_{1}, z_{1}$ and $A_{L i}$, respectively.

The strain energy, $U_{a_{2}}$, in the upper crack arm was written as

$U_{a_{2}}=\Delta a \sum_{i=1}^{i=n_{U}} \iint_{\left(A_{U i}\right)} u_{02 i} d A_{U i}$

where $u_{02 i}$ and $A_{U i}$ are, respectively, the strain energy density and the area of $i$-th layer of upper crack arm (Fig. 3). Also, $y_{3}$ and $z_{3}$ were replaced, respectively, with $y_{2}$ and $z_{2}$ in Eq. 9. Besides, $\varepsilon_{D}$ and $\varepsilon_{K}$ were replaced, respectively, with $\varepsilon_{S_{2}}$ and $\varepsilon_{D_{2}}$. In order to determine $\varepsilon_{S_{2}}$, $\varepsilon_{D_{2}}$ and $\varepsilon_{T}$ Eqs. 10,11 and 12 were modified by replacing of $N, M_{y}, M_{z}, n, y_{3}, z_{3}$ and $A_{i}$ with $N_{2}, M_{y_{2}}, M_{z_{2}}$, $n_{U}, y_{2}, z_{2}$ and $A_{U i}$, respectively. The following equilibrium equations of beam cross-section (Fig. 2 and Fig. $3)$ :

$$
\begin{aligned}
& N_{2}=N-N_{1}, \\
& M_{y_{2}}=M_{y}-M_{y_{1}}+N\left(h_{C_{2}}-h_{C_{3}}\right)-N_{1}\left(h_{C_{2}}-h_{C_{1}}\right), \\
& M_{z_{2}}=M-M_{z_{1}}
\end{aligned}
$$

were used in order to express bending moments, $M_{y_{2}}$ and $M_{z_{2}}$, and the axial force, $N_{2}$, in the upper crack arm in functions of $M_{y}, M_{z}, N$ and $M_{y_{1}}, M_{z_{1}}$ and $N_{1}$.

By substituting of Eqs. 5, 13, 14 and 15 in Eq. 4, we derived

$$
G=\frac{1}{b_{S}}\left(\sum_{i=1}^{i=n_{L}} \iint_{\left(A_{L i}\right)} u_{01 i} d A_{L i}+\sum_{i=1}^{i=n_{U}} \iint_{\left(A_{U i}\right)} u_{02 i} d A_{U i}-\sum_{i=1}^{n} \iint_{\left(A_{i}\right)} u_{0 i} d A_{i}\right)
$$

By Eq. 19, one can calculate the strain energy release rate for delamination cracks in multilayered functionally graded beams by analyzing the strain energy in the beam cross-sections ahead and behind the crack front only. 


\section{Analysis of the strain energy release rate in the multilayered functionally graded ELS beam}

The strain energy release rate in the multilayered functionally graded ELS beam shown in Fig. 4 was analyzed by applying Eq. 19. There is a delamination crack of length, $a$, located arbitrary along the beam height. The lower and upper crack arm thicknesses are $h_{1}$ and $h_{2}$, respectively. The beam has a rectangular cross-section of width, $b$, and height, $2 h$. The beam length is $l$. The external loading consists of a bending moment, $M_{y}$, applied at the free end of lower crack arm (Fig. 4). Thus, the upper crack arm is free of stresses. The beam is clamped in section, $B$. In each layer, the modulus of elasticity varies linearly along the width and thickness of layer. It was assumed also that the moduli of elasticity $E_{H_{i}}, E_{Q_{i}}$ and $E_{R_{i}}$ in points $H_{i}, Q_{i}$ and $R_{i}$ in the $i$-th layer (Fig. 5) vary along the beam length according to the following bi-quadratic laws:

$E_{H_{i}}=E_{H_{0 i}}+\frac{E_{H_{1 i}}-E_{H_{0 i}}}{l^{4}} x^{4}$,

$E_{Q_{i}}=E_{Q_{0 i}}+\frac{E_{Q_{1 i}}-E_{Q_{0 i}}}{l^{4}} x^{4}$

$E_{R_{i}}=E_{R_{0 i}}+\frac{E_{R_{1 i}}-E_{R_{0 i}}}{l^{4}} x^{4}$,

where,

$i=1,2, \ldots, n$,

$0 \leq x \leq l$.

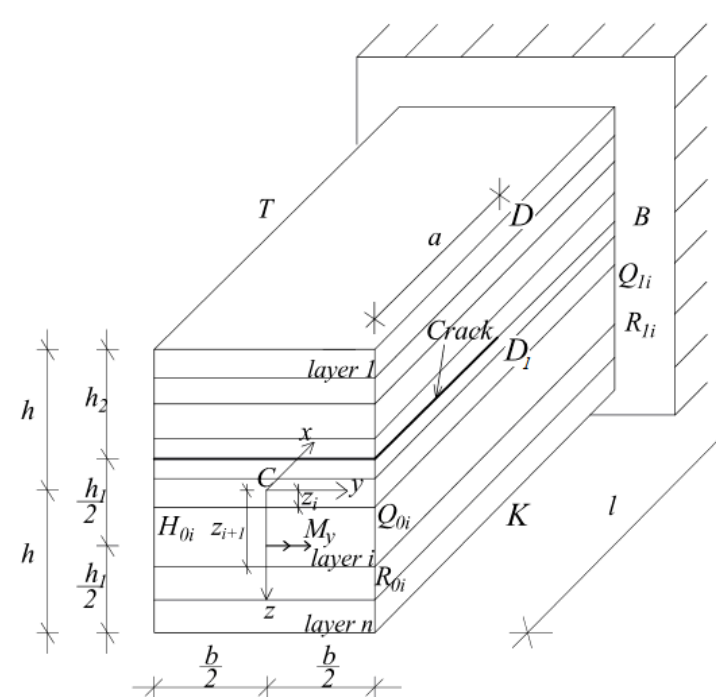

Fig. 4. The functionally graded multilayered ELS beam configuration.

In Eqs. 20, 21 and $22 E_{H_{0 i}}, E_{Q_{0 i}}$ and $E_{R_{0 i}}$ are the moduli of elasticity in points, $H_{0 i}, Q_{0 i}$ and $R_{0 i}$, respectively (Fig. 5). The moduli of elasticity in points, $H_{1 i}, Q_{1 i}$ and $R_{1 i}$, are $E_{H_{1 i}}, E_{Q_{1 i}}$ and $E_{R_{1 i}}$, respectively.

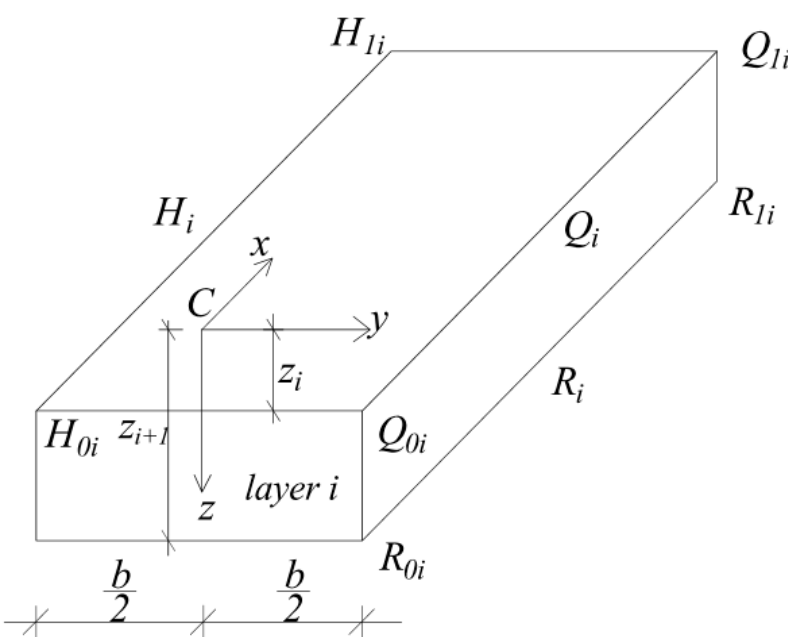

Fig. 5. Notations in the $i$-th layer of the ELS beam.

First, the strain energy density in the beam cross-section, $T D K$, ahead of the crack front was analyzed (Fig. 6). The distribution of modulus of elasticity, $E_{i}$, in the $i$-th layer was obtained by using Eq. 8. For this purpose, by substituting of

$y_{3 H_{i}}=-b / 2, \quad z_{3 H_{i}}=z_{3 i}, y_{3 Q_{i}}=b / 2, \quad z_{3 Q_{i}}=z_{3 i}, \quad y_{3 R_{i}}=b / 2$

and $z_{3 R_{i}}=z_{3 i+1}$ in Eq. 8, we derived

$E_{i}=q_{1 i} y_{3}+q_{2 i} z_{3}+q_{3 i}$,

where

$q_{1 i}=\frac{E_{H_{i}}\left(z_{3 i}-z_{3 i+1}\right)+E_{Q_{i}}\left(z_{3 i+1}-z_{3 i}\right)}{b\left(z_{3 i+1}-z_{3 i}\right)}$,

$q_{2 i}=\frac{E_{R_{i}}-E_{Q_{i}}}{z_{3 i+1}-z_{3 i}}$,

$q_{3 i}=\frac{E_{H_{i}}\left(z_{3 i+1}-z_{3 i}\right)+E_{Q_{i}}\left(z_{3 i+1}+z_{3 i}\right)-2 E_{R_{i}} z_{3 i}}{2\left(z_{3 i+1}-z_{3 i}\right)}$,

$i=1,2, \ldots, n$.

In Eqs. 26, 27 and $28 E_{H_{i}}, E_{Q_{i}}$ and $E_{R_{i}}$ are calculated, respectively, by Eqs. 20, 21 and 22 at $x=a$.

The strain, $\varepsilon$, in the beam cross-section, $T D K$, was expressed by Eq. 9. For this purpose (Fig. 6),

$y_{3 T}=-b / 2, z_{3 T}=-h, y_{3 D}=b / 2, z_{3 D}=h, y_{3 K}=b / 2$ and

$z_{3 K}=h$

were substituted in Eq. 9. In this way, we obtained

$\varepsilon=r_{1} y_{3}+r_{2} z_{3}+r_{3}$,

where 
$r_{1}=\frac{1}{b}\left(\varepsilon_{D}-\varepsilon_{T}\right)$,

$r_{2}=\frac{1}{2 h}\left(\varepsilon_{K}-\varepsilon_{D}\right)$,

$r_{3}=\frac{1}{2}\left(\varepsilon_{K}+\varepsilon_{T}\right)$.

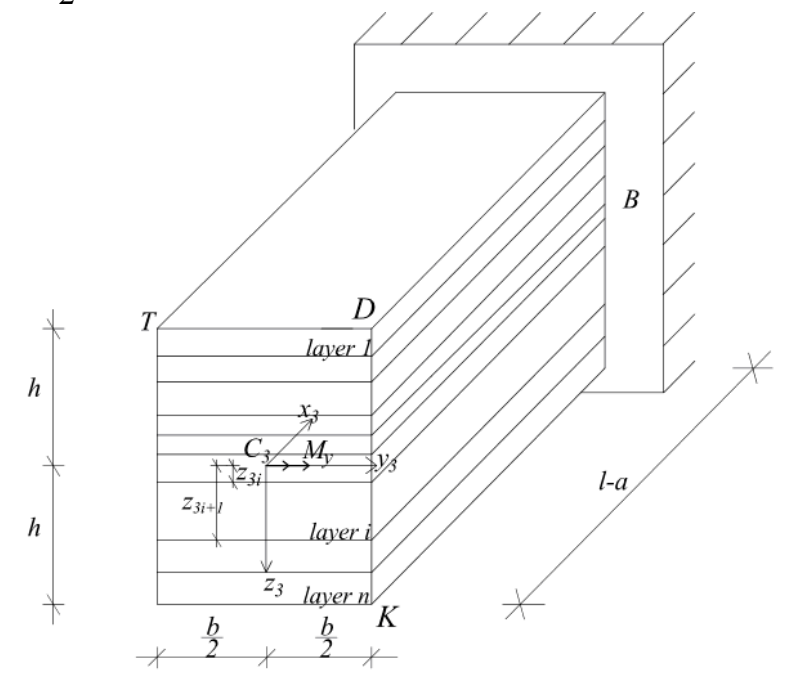

Fig. 6. The un-cracked beam portion ahead of the crack front.

In order to determine the quantities, $r_{1}, r_{2}$ and $r_{3}$, the equilibrium Eqs. 10, 11 and 12, were re-written as

$$
\begin{aligned}
& N=\sum_{i=1}^{i=n} \int_{z_{3 i}}^{z_{3 i+1}}\left(\int_{-\frac{b}{2}}^{\frac{b}{2}} \sigma_{i} d y_{3}\right) d z_{3}, \\
& M_{y_{3}}=\sum_{i=1}^{i=n} \int_{z_{3 i}}^{z_{3 i+1}}\left(\int_{-\frac{b}{2}}^{\frac{b}{2}} \sigma_{i} z_{3} d y_{3}\right) d z_{3}, \\
& M_{z_{3}}=\sum_{i=1}^{i=n} \int_{z_{3 i}}^{z_{3 i+1}}\left(\int_{-\frac{b}{2}}^{\frac{b}{2}} \sigma_{i} y_{3} d y_{3}\right) d z_{3},
\end{aligned}
$$

where (Fig. 6)

$N=0$,

$M_{y_{3}}=M_{y}$,

$M_{z_{3}}=0$.

By substituting of Eqs. 7, 25 and 31 in Eqs. 35, 36 and 37, we obtained

$$
\begin{aligned}
& N=\sum_{i=1}^{i=n}\left[q_{1 i} r_{1} \frac{b^{3}}{12}\left(z_{3 i+1}-z_{3 i}\right)+q_{2 i} r_{2} \frac{b}{3}\left(z_{3 i+1}^{3}-z_{3 i}^{3}\right)+\right. \\
& q_{3 i} r_{2} \frac{b}{2}\left(z_{3 i+1}^{2}-z_{3 i}^{2}\right)+q_{2 i} r_{3} \frac{b}{2}\left(z_{3 i+1}^{2}-z_{3 i}^{2}\right)+ \\
& \left.+q_{3 i} r_{3} b\left(z_{3 i+1}-z_{3 i+1}\right)\right]
\end{aligned},
$$

$$
\begin{aligned}
& M_{y_{3}}=\sum_{i=1}^{i=n}\left[q_{1 i} r_{1} \frac{b^{3}}{24}\left(z_{3 i+1}^{2}-z_{3 i}^{2}\right)+q_{2 i} r_{2} \frac{b}{4}\left(z_{3 i+1}^{4}-z_{3 i}^{4}\right)+\right. \\
& +q_{3 i} r_{2} \frac{b}{3}\left(z_{3 i+1}^{3}-z_{3 i}^{3}\right)+ \\
& \left.+q_{2 i} r_{3} \frac{b}{3}\left(z_{3 i+1}^{3}-z_{3 i}^{3}\right)+q_{3 i} r_{3} \frac{b}{2}\left(z_{3 i+1}^{2}-z_{3 i}^{2}\right)\right] \\
& M_{z_{3}}=\sum_{i=1}^{i=n}\left[q_{2 i} r_{1} \frac{b^{3}}{24}\left(z_{3 i+1}^{2}-z_{3 i}^{2}\right)+q_{3 i} r_{1} \frac{b^{3}}{12}\left(z_{3 i+1}-z_{3 i}\right)+\right. \\
& +q_{1 i} r_{2} \frac{b^{3}}{24}\left(z_{3 i+1}^{2}-z_{3 i}^{2}\right)+ \\
& +q_{1 i} r_{3} \frac{b^{3}}{12}\left(z_{3 i+1}-z_{3 i}\right)
\end{aligned}
$$

The MatLab software can be used to solve Eqs. 41, 42 and 43 with respect to $r_{1}, r_{2}$ and $r_{3}$. Then $r_{1}, r_{2}$ and $r_{3}$ can be substituted in Eq. 31 to obtain the strain distribution in the cross-section of the beam ahead of the crack front.

At $E_{H_{i}}=E_{Q_{i}}=E_{R_{i}}=E$ from Eqs. 31, 41, 42 and 43, we derived

$$
\varepsilon=\frac{3 M_{y_{3}}}{2 b h^{3}} z_{3} .
$$

It should be noted that Eq. 44 is exact match of the formula for strain distribution in a homogeneous beam of rectangular cross-section, $b \times 2 h$, loaded in bending by a moment, $M_{y_{3}}$, [18].

The strain energy densities in the layers ahead of the crack front can be derived by substituting of Eqs. 7, 25 and 31 in Eq. 6.

The analysis of strain energy densities, $u_{01 i}$, in the layers of lower crack arm cross-section, $S_{1} D_{1} K$, behind the crack front (Fig. 7) was carried-out in the following manner. Eqs. 25 and 31 were used to determine the modulus of elasticity and strain distribution, respectively. For this purpose, $y_{3}$, $z_{3}, r_{1}, r_{2}, r_{3}, z_{3 i}, z_{3 i+1}$ and $n$ were replaced, respectively, with $y_{1}, z_{1}, r_{1 L}, r_{2 L}, r_{3 L}, z_{1 i}, z_{1 i+1}$ and $n_{L}$ in Eqs. 25, 31, 41, 42 and 43 . Then the strain energy densities were calculated as

$u_{01 i}=\frac{\sigma_{i}^{2}}{2 E_{i}}$,

where the stress was determined by the Hooke's law. 


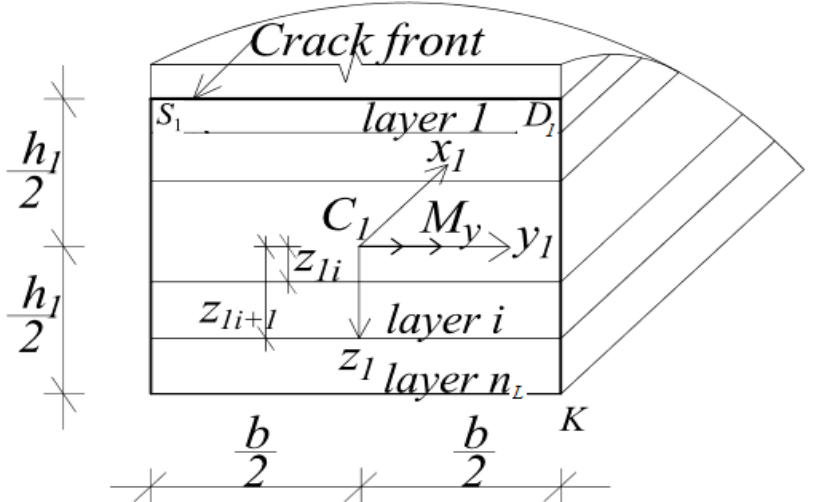

The strain energy density in the upper crack arm is zero. Thus, in view of the fact that for the ELS beam

$$
b_{S}=b
$$

Eq. 19 was re-written as

$$
G=\frac{1}{b}\left(\sum_{i=1}^{i=n_{L}} \int_{z_{1 i}}^{z_{1 i}+\frac{b}{2}} \int_{-\frac{b}{2}}^{\frac{b}{2}} u_{01 i} d y_{1} d z_{1}-\sum_{i=1}^{i=n} \int_{z_{3 i}}^{z_{3 i+1}} \int_{-\frac{b}{2}}^{\frac{b}{2}} u_{0 i} d y_{3} d z_{3}\right) .
$$

Fig. 7. The lower crack arm cross-section behind the crack front.

$$
\begin{aligned}
G & =\sum_{i=1}^{i=n_{L}}\left[r_{1 L} r_{2 L} q_{1 i} \frac{b^{2}}{24}\left(z_{1 i+1}^{2}-z_{1 i}^{2}\right)+r_{1 L} r_{3 L} q_{1 i} \frac{b^{2}}{12}\left(z_{1 i+1}-z_{1 i}\right)+r_{1 L}^{2} q_{2 i} \frac{b^{2}}{48}\left(z_{1 i+1}^{2}-z_{1 i}^{2}\right)+\right. \\
+ & \frac{1}{8} r_{2 L}^{2} q_{2 i}\left(z_{1 i+1}^{4}-z_{1 i}^{4}\right)+\frac{1}{4} r_{3 L}^{2} q_{2 i}\left(z_{1 i+1}^{2}-z_{1 i}^{2}\right)+\frac{1}{3} r_{1 L} r_{3 L} q_{2 i}\left(z_{1 i+1}^{3}-z_{1 i}^{3}\right)+ \\
+ & r_{1 L}^{2} q_{3 i} \frac{b^{2}}{24}\left(z_{1 i+1}-z_{1 i}\right)+\frac{1}{6} r_{2 L}^{2} q_{3 i}\left(z_{1 i+1}^{3}-z_{1 i}^{3}\right)+\frac{1}{2} r_{3 L}^{2} q_{3 i}\left(z_{1 i+1}-z_{1 i}\right)+ \\
+ & \left.\frac{1}{2} r_{1 L} r_{3 L} q_{3 i}\left(z_{1 i+1}^{2}-z_{1 i}^{2}\right)\right]- \\
& -\sum_{i=1}^{i=n}\left[r_{1} r_{2} q_{1 i} \frac{b^{2}}{24}\left(z_{3 i+1}^{2}-z_{3 i}^{2}\right)+r_{1} r_{3} q_{1 i} \frac{b^{2}}{12}\left(z_{3 i+1}-z_{3 i}\right)+r_{1}^{2} q_{2 i} \frac{b^{2}}{48}\left(z_{3 i+1}^{2}-z_{3 i}^{2}\right)+\right. \\
& +\frac{1}{8} r_{2}^{2} q_{2 i}\left(z_{3 i+1}^{4}-z_{3 i}^{4}\right)+\frac{1}{4} r_{3}^{2} q_{2 i}\left(z_{3 i+1}^{2}-z_{3 i}^{2}\right)+\frac{1}{3} r_{1} r_{3} q_{2 i}\left(z_{3 i+1}^{3}-z_{3 i}^{3}\right)+ \\
& +r_{1}^{2} q_{3 i} \frac{b^{2}}{24}\left(z_{3 i+1}-z_{3 i}\right)+\frac{1}{6} r_{2}^{2} q_{3 i}\left(z_{3 i+1}^{3}-z_{3 i}^{3}\right)+\frac{1}{2} r_{3}^{2} q_{3 i}\left(z_{3 i+1}-z_{3 i}\right)+ \\
& +\frac{1}{2} r_{1} r_{3 l} q_{3 i}\left(z_{3 i+1}^{2}-z_{3 i}^{2}\right)
\end{aligned}
$$

Eq. 48 determines the strain energy release rate in the multilayered functionally graded ELS beam configuration

By substituting of $n=n_{L}=1, \quad h_{1}=h \quad$ and $E_{H_{i}}=E_{Q_{i}}=E_{R_{i}}=E$ in Eq. 48, we derived

$G=\frac{21 M_{y}^{2}}{4 E h^{3}}$,

which is exact match of the formula for strain energy release rate when the ELS beam is homogeneous and the delamination crack is located in the beam mid-plane [16].

An additional analysis of the strain energy release rate in the multilayered functionally graded ELS beam was performed for verification. For this purpose, the fact [19] that for linear-elastic materials the strain energy release rate can obtained by differentiating the beam strain energy, $U$, with respect to the crack area, $A_{a}$, was used

$G=\frac{d U}{d A_{a}}$

where

$$
d A_{a}=b d a .
$$

In Eq. 51, $d a$ is an elementary increase of the crack length. By combining of Eqs. 50 and 51, we obtained

$$
G=\frac{d U}{b d a}
$$

The multilayered functionally graded ELS beam strain energy was derived by integration of the strain energy densities in the lower crack arm (the upper crack is free of stresses) and in the un-cracked beam portion

$$
\begin{aligned}
& U=\sum_{i=1}^{i=n_{L}} \int_{z_{1 i}}^{z_{1 i+1}}\left[\int_{-\frac{b}{2}}^{\frac{b}{2}}\left(\int_{0}^{a} u_{01 i} d x\right) d y_{1}\right] d z_{1}+ \\
& \sum_{i=1}^{i=n} \int_{z_{3 i}}^{z_{3 i+1}}\left[\int_{-\frac{b}{2}}^{\frac{b}{2}}\left(\int_{a}^{l} u_{0 i} d x\right) d y_{3}\right] d z_{3},
\end{aligned}
$$

where $x$-axis is shown in Fig. 4. After substituting the strain energy densities, $u_{01 i}$ and $u_{0 i}$, in Eq. 53 and performing the 
differentiation in Eq. 52, we obtained strain energy release rate that is exact match of Eq. 48. This fact verifies the strain energy release rate analysis developed in the present paper. It should be noted that $G$ was calculated relatively simply by using Eq. 52 for the multilayered functionally graded ELS beam configuration in Fig. 4. However, for more complicated structures and loading conditions Eq. 19 has decisive advantages over Eq. 52. For instance, by applying Eq. 19, one can obtain the strain energy release rate by determining the strain energy in the beam cross-sections ahead and behind the crack front only in contrast to Eq. 52 which requires analysis of the whole structure.

The dependences of delamination fracture behaviour of the three-dimensional functionally graded multilayered ELS beam configuration on the material gradient were elucidated. For this purpose, calculations of the strain energy release rate were carried-out by using Eq. 48. In order to get specific results, two three-layered ELS beams were analyzed. In the beam in Fig. 8a, the delamination crack is located between layers 2 and 3. The delamination crack in the configuration in Fig. $8 \mathrm{~b}$ is between layers 1 and 2. In both configurations, the layer thickness is $t_{l}$ (Fig. 8). In the calculations, it was assumed that $t_{l}=0.002 \mathrm{~m}, b=0.02 \mathrm{~m}$ and $M_{y}=10 \mathrm{Nm}$. The strain energy release rate was obtained in dimensionless form by using the formula, $G_{N}=G /\left(E_{R_{01}} b\right)$. The strain energy release as a function of $E_{R_{03}} / E_{R_{01}}$ ratio for the two three-layered ELS configurations at $E_{Q_{01}} / E_{R_{01}}=0.5$, $E_{H_{01}} / E_{R_{01}}=2, \quad E_{R_{11}} / E_{R_{01}}=2, \quad E_{Q_{11}} / E_{Q_{01}}=2$, $E_{H_{11}} / E_{H_{01}}=2, \quad E_{R_{02}} / E_{R_{01}}=2, \quad E_{Q_{02}} / E_{R_{02}}=1$, $E_{H_{02}} / E_{R_{02}}=0.5, \quad E_{R_{12}} / E_{R_{02}}=2, \quad E_{Q_{12}} / E_{Q_{02}}=2$, $E_{H_{12}} / E_{H_{02}}=2, \quad E_{Q_{03}} / E_{R_{03}}=2, \quad E_{H_{03}} / E_{R_{03}}=1$, $E_{R_{13}} / E_{R_{03}}=2, E_{Q_{13}} / E_{Q_{03}}=2, E_{H_{13}} / E_{H_{03}}=2$ and $a / l=0.5$ is shown in Fig. 9. It should be noted that $E_{R_{01}}$ was kept constant in the calculations. Thus, $E_{R_{03}}$ was varied in order to generate various $E_{R_{03}} / E_{R_{01}}$ ratios. The diagrams in Fig. 9 show that the strain energy release rate decreases with increasing of $E_{R_{03}} / E_{R_{01}}$ ratio (this behaviour is due to the increase of beam stiffness). One can also observe in Fig. 9 that when the crack is located between layers 2 and 3, the strain energy release rate is higher in comparison with the case when the crack is between layers 1 and 2. This finding was attributed to the fact that the lower crack arm is thicker when the crack is between layers 1 and 2 (the upper crack arm is free of stresses).

The dependence of strain energy release rate on the length of the crack was elucidated too. The ELS beam in Fig. $8 \mathrm{~b}$ was considered. The crack length was characterized by $a / l$ ratio. The strain energy release rate in dimensionless form as a function of $a / l$ ratio for three $E_{R_{02}} / E_{R_{01}}$ ratios at

$$
\begin{array}{lll}
E_{Q_{01}} / E_{R_{01}}=0.5, & E_{H_{01}} / E_{R_{01}}=2, & E_{R_{11}} / E_{R_{01}}=2, \\
E_{Q_{11}} / E_{Q_{01}}=2, & E_{H_{11}} / E_{H_{01}}=2, & E_{Q_{02}} / E_{R_{02}}=1, \\
E_{H_{02}} / E_{R_{02}}=0.5, & E_{R_{12}} / E_{R_{02}}=2, & E_{Q_{12}} / E_{Q_{02}}=2,
\end{array}
$$

$E_{H_{12}} / E_{H_{02}}=2$,

$$
E_{R_{03}} / E_{R_{01}}=0.5,
$$$$
E_{Q_{03}} / E_{R_{03}}=2,
$$

$E_{H_{03}} / E_{R_{03}}=1$,

$$
E_{R_{13}} / E_{R_{03}}=2 \text {, }
$$$$
E_{Q_{13}} / E_{Q_{03}}=2
$$

$E_{H_{13}} / E_{H_{03}}=2$ was presented in Fig. 10. Fig. 10 shows that increase of $a / l$ ratio leads to decrease of the strain energy release (this can be explained by the fact that the modulus of elasticity in the beam cross-section in which the crack front is located increases with increasing of the crack length, since the modulus of elasticity in the beam clamped end are higher than in the beam free end). Also, it can be observed in Fig. 10 that the strain energy release rate decreases when $E_{R_{02}} / E_{R_{01}}$ ratio increases (this behaviour should be attributed to the increase of beam stiffness).
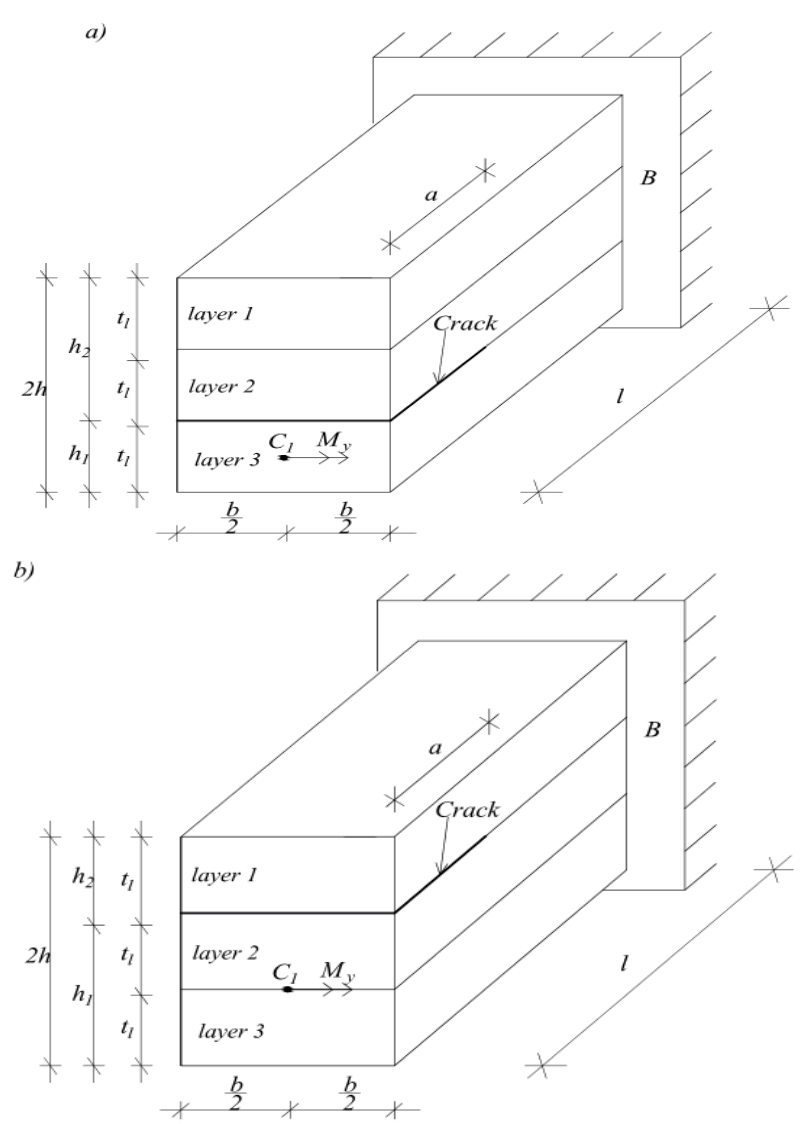

Fig. 8. Two three-layered ELS beam configurations.

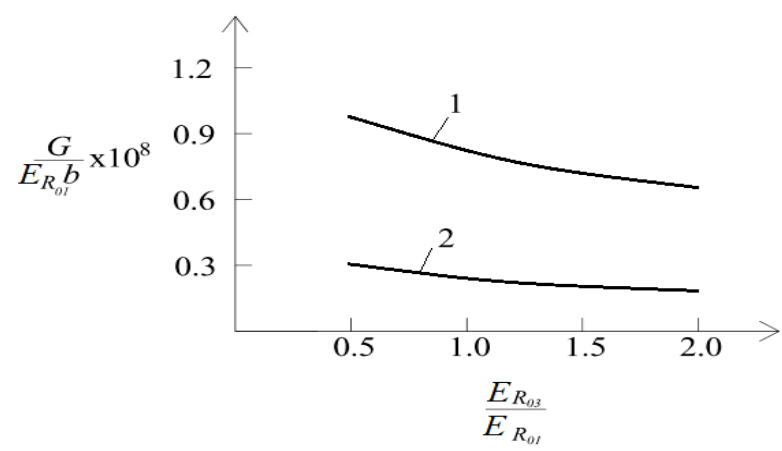

Fig. 9. The strain energy release rate in dimensionless form presented as a function of $E_{R_{03}} / E_{R_{01}}$ ratio (curve 1 - for crack between layers 2 and 3 , curve 2 - for crack between layers 1 and 2 (refer to Fig. 8)). 


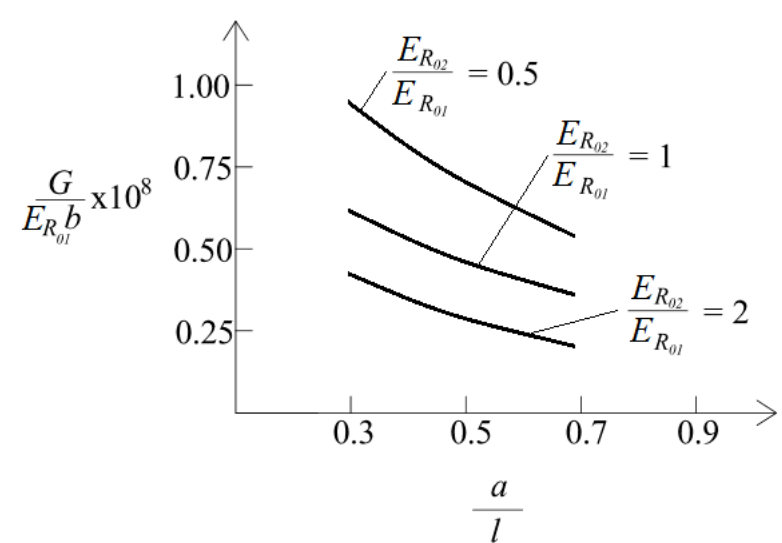

Fig. 10. The strain energy release rate in dimensionless form presented as a function of $a / l$ ratio at three $E_{R_{02}} / E_{R_{01}}$ ratios.

\section{Conclusions}

Delamination fracture in three-dimensional functionally graded multilayered linear-elastic beam configurations loaded by bending moments and axial forces was studied. An analytical solution to the strain energy release rate was obtained. Beam cross-sections with one axis of symmetry were analyzed. The delamination crack can be located arbitrary between layers. The solution derived is valid for beams with any number of layers of different thicknesses and material properties. The material is functionally graded in width, thickness and length directions. Linear variation of the modulus of elasticity in width and thickness directions was assumed. Along the layer length, the modulus of elasticity can vary arbitrary. The bending moments and axial forces in the beam cross-sections ahead and behind the crack front were used to calculate the strain energy release rate. The solution derived was used to investigate delamination fracture in the three-dimensional functionally graded ELS beam. A quadratic law was adopted to describe the distribution of modulus of elasticity in beam length direction. Specific results were obtained for two threelayered ELS beam configurations. The analysis indicated that increase of $E_{R_{03}} / E_{R_{01}}$ and $E_{R_{02}} / E_{R_{01}}$ ratios lead to decrease of the strain energy release rate. Also, it was found that increase of the crack length leads to decrease of the strain energy release rate when the moduli of elasticity in the beam clamped end are higher than in the beam free end.

\section{Acknowledgments}

The financial support provided by the Research and Design Centre (CNIP) of the UACEG, Sofia (Contract BN 189/2016) is greatly appreciated.

This is an Open Access article distributed under the terms of the Creative Commons Attribution Licence

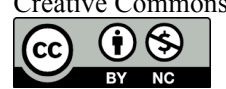

\section{References}

1. Markworth, A.J., Ramesh, K.S., Parks, Jr.W.P., "Review: modelling studies applied to functionally graded materials", Journal of Material Sciences, 30(3), 1995, pp. 2183-2193.

2. Suresh, S., Mortensen, A., "Fundamentals of functionally graded materials", London: IOM Communications Ltd, 1998.

3. Hirai, T., Chen, L., "Recent and prospective development of functionally graded materials in Japan", Mater Science Forum, 308311, 1999, pp. 509-514.

4. Gasik, M. M., "Functionally graded materials: bulk processing techniques", International Journal of Materials and Product Technology, 39(2), 2010, pp. 20-29.

5. Nemat-Allal, M. M., Ata, M. H., Bayoumi, M. R., Khair-Eldeen, W., "Powder metallurgical fabrication and microstructural investigations of Aluminum/Steel functionally graded material”, Materials Sciences and Applications, 2(3), 2011, pp. 1708-1718.

6. Bohidar, S. K., Sharma, R., Mishra, P. R., "Functionally graded materials: A critical review", International Journal of Research, 1(4), 2014, pp. 289-301.

7. Szekrenyes, A., "Semi-layerwise analysis of laminated plates with nonsingular delamination -The theorem of autocontinuity", Applied mathematical modelling, 40(2), 2016, pp. 1344 - 1371.

8. Szekrenyes, A., "Nonsingular crack modelling in orthotropic plates by four equivalent single layers", European journal of mechanics A/solids, 55(2), 2016, pp. 73-99.

9. Tkacheva, L.A., "Unsteady crack propagation in the beam approximation", Applied Mechanics and Technical Physics, 49(4), 2008 , pp. $177-189$.
10. Erdogan, F., "Fracture mechanics of functionally graded materials", Composite Engineering, 5(2), 1995, pp. 753-770.

11. Paulino, G. C., "Fracture in functionally graded materials", Engineering Fracture Mechanics, 69(3), 2002, pp. 1519-1530.

12. Tilbrook, M. T., Moon, R. J., Hoffman, M., "Crack propagation in graded composites", Composite Science and Technology, 65(1), 2005, pp. 201-220.

13. Carpinteri, A., Pugno, N., "Cracks in re-entrant corners in functionally graded materials", Engineering Fracture Mechanics, 73(1), 2006, pp. 1279-1291.

14. Upadhyay, A. K., Simha, K. R. Y., "Equivalent homogeneous variable depth beams for cracked FGM beams; compliance approach", International Journal of Fracture, 144(4), 2007, pp. 209-213.

15. Shi-Dong Pan, Ji-Cai Feng, Zhen-Gong Zhou, Wu-Lin-Zhi, "Four parallel non-symetric Mode -III cracks with different lengths in a functionally graded material plane", Strength, Fracture and Complexity: an International Journal, 5(3), 2009, pp. 143-166.

16. Hutchinson, J. W., Suo, Z., "Mixed mode cracking in layered materials", Advances in Applied Mechanics, 64(2), 1992, pp. 804810 .

17. Korn, G., Korn, T., "Mathematical handbook for scientists and engineers", Nauka, M., 1970.

18. Dowling, N., "Mechanical Behavior of Materials", Pearson, 2007.

19. Parton, V.Z., "Fracture mechanics, Nauka", M., 1990. 